\title{
BMJ Open Determining the optimal time for liberation from renal replacement therapy in critically ill patients: protocol for a systematic review and meta- analysis (DOnE RRT)
}

\author{
Abdalrhman Al Saadon, ${ }^{1}$ Riley Katulka, ${ }^{1}$ Meghan Sebastianski, ${ }^{2}$ \\ Robin Featherstone, ${ }^{2,3}$ Ben Vandermeer, ${ }^{3} \mathrm{R}$ T Noel Gibney, ${ }^{1}$ Oleksa G Rewa, \\ Sean M Bagshaw ${ }^{1}$
}

To cite: Al Saadon A, Katulka R, Sebastianski M, et al. Determining the optimal time for liberation from renal replacement therapy in critically ill patients: protocol for a systematic review and metaanalysis (DOnE RRT). BMJ Open 2018;8:e23306. doi:10.1136/ bmjopen-2018-023306

- Prepublication history and additional material for this paper are available online. To view these files, please visit the journal online (http://dx.doi. org/10.1136/bmjopen-2018023306).

Received 21 April 2018 Revised 10 August 2018 Accepted 9 October 2018

Check for updates

(C) Author(s) (or their employer(s)) 2018. Re-use permitted under CC BY-NC. No commercial re-use. See rights and permissions. Published by BMJ.

For numbered affiliations see end of article.

Correspondence to Dr Sean M Bagshaw; bagshaw@ualberta.ca

\section{ABSTRACT}

Introduction Renal replacement therapy (RRT) is a complex and expensive form of life-sustaining therapy, reserved for our most acutely ill patients. While a number of randomised trials have evaluated the optimal timing to start RRT among critically ill patients in the intensive care unit (ICU), there has been a paucity of trials providing guidance on when and under what circumstances to ideally liberate a patient from RRT. We are conducting a systematic review and meta-analysis to identify clinical and biochemical markers that predict kidney recovery and successful liberation from acute RRT among critically ill patients with acute kidney injury.

Methods and analysis Our comprehensive search strategy was developed in consultation with a research librarian and independently peer-reviewed by a second librarian. We will search electronic databases: Ovid Medline, Ovid Embase and Wiley Cochrane Library. Selected grey literature sources will also be searched. Our search strategies will focus on concepts related to RRT (ie, intermittent haemodialysis, slow low-efficiency dialysis, continuous renal replacement therapy), intensive care (ie, involving any ICU setting) and discontinuation of therapy (ie, either clinical, physiological and biochemical parameters of weaning acute RRT) from 1990 to October 10, 2017. Citation screening, selection, quality assessment and data abstraction will be performed in duplicate. Studies will, where possible, be pooled in statistical meta-analysis. When deemed sufficiently clinically homogenous, and we have four or more studies reporting, sensitivities and specificities will be pooled simultaneously using a hierarchical summary receiver operator characteristic curve and bivariate analysis.

Ethics and dissemination Our systematic review will synthesise the literature on clinical and biochemical markers that predict liberation from RRT. Research ethics approval is not required.

Trial registration number CRD42018074615.
Strengths and limitations of this study

- There are relatively few predictors of successful weaning studied, and similar parameters may be defined heterogeneously between studies which limits our ability to perform a pooled analysis.

- Our search strategy will include studies from 1990 to present, which may result in bias due to significant changes in the management of acute renal replacement therapy (RRT) over the time period included.

- Mortality will not be included specifically in our search terms, which may result in studies with mortality as an endpoint not being identified.

- Studies evaluating peritoneal dialysis as a form of acute RRT were omitted due to the limited use of this modality in developed countries; however, this may limit generalisability of results in regions where this modality is more commonly practised.

- We anticipate variable study methodology and quality of reporting across the retrieved studies, which may compromise confidence in inferences from the review. Furthermore, data from case series cannot be pooled in meta-analysis as the lack of a control group precludes the construction of $2 \times 2$ tables.

\section{BACKGROUND}

Acute kidney injury (AKI) is a common problem in critically ill patients. It is estimated to occur in $6 \%-70 \%$ of all intensive care unit (ICU) patients depending on the definition used. ${ }^{1-3}$ When AKI progresses to overt renal failure, renal replacement therapy (RRT) is often necessary. The incidence of RRT treated AKI in the ICU is $13 \%$, with continuous renal replacement therapy (CRRT) being used in $75 \%$ of cases. ${ }^{4}$

RRT is a complex and expensive form of life-sustaining therapy, reserved for our most acutely ill patients. Because its provision can be associated with complications, including 
catheter-associated infections, clinically significant haemorrhage, haemodynamic instability and delayed renal recovery, ${ }^{5-12}$ it is imperative to determine the optimal time to liberate patients from this therapy.

While a number of randomised trials have evaluated the timing of initiation of RRT among critically ill patients, there has been a paucity of trials providing guidance on when and under what circumstances to ideally liberate a patient from acute RRT. ${ }^{13-15}$ The Kidney Disease Improving Global Outcomes (KDIGO) organisation has stated in their 2012 Clinical Practice Guidelines for Acute Kidney Injury to, "discontinue RRT when it is no longer required, either because intrinsic kidney function has recovered to the point that it is adequate to meet patient needs, or because RRT is no longer consistent with the goals of care." ${ }^{16}$ However, this recommendation was largely based on expert opinion.

Although no specific criteria have rigorous evidence to support how best to determine when kidney function has sufficiently recovered to warrant a trial of liberation from acute RRT, ${ }^{17}$ several clinical parameters have been evaluated, including urine output before and after cessation of RRT, creatinine clearance, daily urinary creatinine ${ }^{18}$ kinetic estimated glomerular filtration rate (eGFR), and urea excretion. ${ }^{19}$ Of these, urine output is the most-studied and has been shown to have good predictive value. Hence, its use as a marker to wean RRT in two recent major trials. ${ }^{1415}$ In addition to these clinical parameters, biomarkers such as neutrophil gelatinase-associated lipocalin (NGAL) and cystatin $\mathrm{C}$ (CysC) have been proposed as better predictors of renal function than serum creatinine in patients with $\mathrm{AKI},{ }^{20-22}$ and recent studies have shown promise in using CysC specifically to guide weaning from RRT. ${ }^{23-25}$ Although many parameters and markers of renal recovery have been evaluated to guide cessation of RRT, the available data have not yet been rigorously synthesised.

Accordingly, we will conduct a systematic review and meta-analysis to identify clinical and biochemical markers that predict kidney recovery and successful liberation from acute RRT among critically ill patients with AKI. This is a vital initial step towards identifying, validating and implementing evidence-informed parameters to guide best practice, optimise RRT utilisation, improve healthcare economics and ultimately improve outcomes of critically ill patients receiving acute RRT.

\section{METHODS}

\section{Patient and public involvement}

No patient or public were involved in this systematic review and meta-analysis.

\section{Study design}

A systematic review will be performed using guidelines from The Cochrane Collaboration and Center for reviews and dissemination and described according to
Preferred Reporting Items for Systematic Reviews and Meta-Analyses Protocol (PRISMA-P) guidelines (http:// www.systematicreviewsjournal.com/content/4/1/1). ${ }^{26} 27$

\section{Study registration}

In accordance with PRISMA-P guidelines, our systematic review is registered with the International Prospective Register of Systematic Reviews.

\section{Data source and search methods}

The search strategy will be developed and performed in consultation with a research librarian and independently peer-reviewed by a second librarian. ${ }^{28}$ We will search electronic databases: Ovid Medline (1946-), Ovid Embase (1988-) and Wiley Cochrane Library (inception-). Our search strategy will combine concepts related to RRT (ie, intermittent haemodialysis (IHD), slow low-efficiency dialysis (SLED), CRRT), intensive care (ie, involving any ICU setting) and discontinuation of therapy (ie, either clinical, physiological and biochemical parameters of weaning acute RRT) or treatment outcome. We will limit search results to publications from 1990 to October 10, 2017. No language limits will be applied. See online supplementary appendix A for the Medline strategy.

Additional search sources will include the trial registry platforms (ie, ClinicalTrials.gov) and Google Scholar. We will also search for meeting abstracts from the past 2 years, where available, using Conference Proceedings Citation Index (Clarivate Analytics), and by hand-searching published proceedings from the following associations and meetings: American Society of Nephrology, Canadian Society of Nephrology, 'CRRTonline' (San Diego), European Renal Association - European Dialysis and Transplant Association, European Society of Intensive Care Medicine, International Symposium on Intensive Care and Emergency Medicine (Brussels), National Kidney Foundation, and Society of Critical Care Medicine. Search results will be exported and screened in EndNote V.X7 (Clarivate Analytics).

\section{Study selection}

Eligible articles will be identified through two phases. In the first phase, two authors (AA and RK) will independently review the titles and abstracts of all retrieved articles and documents using EndNote V.X7 (Thomson Reuters, Philadelphia, Pennsylvania, USA) for potential inclusion. Disagreements will be resolved through discussion or adjudication by a third author (OGR). In the second phase, full texts of the selected articles will be reviewed by the same two authors independently and reviewed for eligibility using standard, pre-defined criteria. Disagreements will be resolved through discussion with a third author (OGR). Eligible studies will have the following criteria.

\section{Eligibility criteria}

Studies will be included if they mention all of the following themes: (1) intensive care (ie, intended to refer to patients supported in an ICU setting capable 
of providing invasive mechanical ventilation or vasoactive therapy); (2) RRT (ie, IHD, SLED, CRRT) and (3) describe parameters associated with weaning or liberation (ie, clinical, physiological and biochemical parameters). We will consider those studies published after 1990 and levels of evidence which include original data (ie, randomised control trials, cohort studies, case-control studies, case series). We will review published secondary uses of data if not already included and consider existing evidence syntheses (ie, systematic reviews, meta-analyses and Cochrane reviews), as well as targeted grey literature (ie, conference abstracts and proceedings).

\section{Exclusion criteria}

Studies will be excluded that do not fulfil all of the above criteria.

\section{Outcome measures}

The primary outcome will be 'successful discontinuation of RRT' among patients receiving RRT, however defined by the study. Secondary outcomes will include description of the spectrum of definitions for 'successful discontinuation of RRT', duration of RRT, new chronic kidney disease or RRT utilisation after ICU discharge.

\section{Data extraction}

A structured data extraction tool will be developed and piloted to extract study-specific data. Data will be extracted by two authors independently (AA and RK). Data abstracted will include publication details, study design, operational definition, patient characteristics, RRT discontinuation parameter details, the timing of measurement of markers of RRT weaning and primary and secondary outcomes of interest. Disagreements will be resolved through discussion or adjudication by a third author (OGR). The authors of the retrieved studies and/ or documents will be contacted for further information if necessary.

\section{Risk of bias assessment}

Study methodological quality will be independently rated by two authors (AA and RK) using the for Newcastle Ottawa Scale for observational studies and the Cochrane Collaboration's tool for randomised controlled trials. ${ }^{29} 30$ The strength of outcome measures derived from each study will be evaluated using the Grading of Recommedations, Assessment, Development and Evaluations (GRADE) system (clinicalevidence.bmj.com/x/set/ static/ebm/learn/665072.html) and the MOOSE checklist for meta-analysis and systematic reviews of observational studies. ${ }^{31}$

\section{Data analysis}

Studies will, where possible, be pooled in statistical meta-analysis. $2 \times 2$ tables will be constructed from exposures and outcomes of weaning parameters reported in each study where available. If not reported, we will contact authors for the data and if necessary calculate missing statistical parameters from reported values. Likelihood ratios will be reported in addition to sensitivities and specificities. If adjusted and unadjusted results are presented, unadjusted data will preferentially be used for meta-analysis although adjusted data will be presented where given. Where results are reported as odds ratios and it is not possible to construct $2 \times 2$ tables from the available data, they will not be included in the statistical meta-analysis.

We will use random effects model to pool effect sizes for each exposure as it associates with the primary outcome. Dichotomous outcomes will be reported as pooled odd ratios and 95\% CIs based on the DerSimonian-Laird random-effect model. Continuous outcomes will be reported using calculated weighted mean differences with their $95 \%$ CIs. Using the $\mathrm{I}^{2}$ statistic and $\chi^{2}$ test, we will assess heterogeneity among studies. The thresholds for interpretation of $\mathrm{I}^{2}$ will be in accordance with the definitions presented in the Cochrane Handbook for Systematic Reviews of Interventions. ${ }^{26}$

When deemed sufficiently clinically homogenous, and we have four or more studies reporting, sensitivities and specificities will be pooled simultaneously using a hierarchical summary receiver operator characteristic (HSROC) curve and bivariate analysis. ${ }^{32}$ Pooled, joint estimates of sensitivity and specificity as well as the HSROC curve will be presented from these analyses.

\section{Subgroup analysis}

A planned subgroup analysis will include different RRT modalities providing such data are available: CRRT, intermittent renal replacement therapy (include both IHD and SLED), age stratified by $\geq 60$ and $<60$ years old and chronic kidney disease (CKD) status prior to RRT, defined by eGFR $<60$ and across KDIGO stages of CKD as study permits. A $\chi^{2}$ test will be used to specify subgroup effect.

We will also perform a subgroup analysis comparing older (1990-2007) to newer studies (2008-current) in order to assess for possible bias resulting from significant changes to the management of severe AKI over the time period of the retrieved publications.

\section{Ethics and dissemination}

Our systematic review will synthesise the literature on clinical and biochemical markers that predict liberation from acute RRT. The results will be presented at national as well as international conferences in poster or oral presentations. The final manuscript will be published in a peer-reviewed journal. The pooled analysis of this review will be used to update clinical practice guidelines on management of RRT in AKI. ${ }^{16}$

\section{CONCLUSION}

Our systematic review and meta-analysis aims to identify clinical, physiological and biochemical markers that predict kidney recovery and successful liberation from acute RRT and will inform on the optimum utilisation of RRT and improve outcomes for patients receiving RRT. 
We anticipate our systematic review will inform future evidence-based clinical practice guidelines on the optimal circumstances to discontinue RRT in critically ill patients.

\section{Author affiliations}

${ }^{1}$ Department of Critical Care Medicine, Faculty of Medicine and Dentistry, University of Alberta, Edmonton, Alberta, Canada

${ }^{2}$ Alberta Strategy for Patient Oriented Research (SPOR) SUPPORT Unit Knowledge Translation Platform, University of Alberta, Edmonton, Alberta, Canada ${ }^{3}$ Alberta Research Center for Health Evidence (ARCHE), University of Alberta, Edmonton, Alberta, Canada

Acknowledgements SMB is supported by a Canada Research Chair in Critica Care Nephrology. Research support for this project was received by the Alberta Strategy for Patient Orientated Research (SPOR) SUPPORT Unit Knowledge Translation Platform. The authors acknowledge Tara Landry, MLIS, for her peerreview of the search strategy.

Contributors SMB conceived the study. AAS and OGR drafted the manuscript. RK provided screening and data abstraction. RF created the search strategy. BV provided the statistical analysis in the protocol. RTNG and MS reviewed the manuscript and provided comments. SMB is the guarantor.

Funding The Alberta Strategy for Patient Oriented Research (SPOR) SUPPORT unit was the sole sponsor for this study.

Competing interests SMB and OGR has consulted for and received grant support from Baxter Healthcare Corp.

Patient consent Not required.

Provenance and peer review Not commissioned; externally peer reviewed.

Open access This is an open access article distributed in accordance with the Creative Commons Attribution Non Commercial (CC BY-NC 4.0) license, which permits others to distribute, remix, adapt, build upon this work non-commercially, and license their derivative works on different terms, provided the original work is properly cited, appropriate credit is given, any changes made indicated, and the use is non-commercial. See: http://creativecommons.org/licenses/by-nc/4.0/.

\section{REFERENCES}

1. Cruz DN, Bolgan I, Perazella MA, et al. North East Italian Prospective Hospital Renal Outcome Survey on Acute Kidney Injury (NEiPHROSAKI): targeting the problem with the RIFLE Criteria. Clin J Am Soc Nephrol 2007;2:418-25.

2. Hoste EA, Clermont G, Kersten A, et al. RIFLE criteria for acute kidney injury are associated with hospital mortality in critically ill patients: a cohort analysis. Crit Care 2006;10:R73.

3. Laake JH, Bugge JF. Acute renal failure in critically ill patients. Tidsskrift for den Norske lægeforening: tidsskrift for praktisk medicin, ny række 2010;130:158.

4. Hoste EA, Bagshaw SM, Bellomo R, et al. Epidemiology of acute kidney injury in critically ill patients: the multinational AKI-EPI study. Intensive Care Med 2015;41:1411-23.

5. Ferreira JA, Johnson DW. The incidence of thrombocytopenia associated with continuous renal replacement therapy in critically ill patients. Ren Fail 2015;37:1232-6.

6. Guru PK, Singh TD, Akhoundi A, et al. Association of thrombocytopenia and mortality in critically III patients on continuous renal replacement therapy. Nephron 2016;133:175-82.

7. Hoste EA, Blot SI, Lameire NH, et al. Effect of nosocomial bloodstream infection on the outcome of critically ill patients with acute renal failure treated with renal replacement therapy. J Am Soc Nephrol 2004;15:454-62.

8. Ronco C, Bellomo R. Complications with continuous renal replacement therapy. American Journal of Kidney Diseases 1996;28:S100-S104.
9. Silversides JA, Pinto R, Kuint R, et al. Fluid balance, intradialytic hypotension, and outcomes in critically ill patients undergoing renal replacement therapy: a cohort study. Crit Care 2014;18:624.

10. van de Wetering J, Westendorp RG, van der Hoeven JG, et al Heparin use in continuous renal replacement procedures: the struggle between filter coagulation and patient hemorrhage. J Am Soc Nephrol 1996;7:145.

11. Cerdá J, Liu KD, Cruz DN, et al. Promoting kidney function recovery in patients with AKI requiring RRT. Clin J Am Soc Nephrol 2015;10:1859-67.

12. Schiffl $H$. Renal recovery after severe acute renal injury. Eur J Med Res 2008;13:552.

13. Smith OM, Wald R, Adhikari NK, et al. Standard versus accelerated initiation of renal replacement therapy in acute kidney injury (STARRT-AKI): study protocol for a randomized controlled trial. Trials 2013;14:320.

14. Gaudry S, Hajage D, Schortgen F, et al. Initiation strategies for renal-replacement therapy in the intensive care unit. $N$ Engl J Med 2016;375:122-33.

15. Zarbock A, Kellum JA, Schmidt C, et al. Effect of early vs delayed initiation of renal replacement therapy on mortality in critically ill patients with acute kidney injury: the ELAIN randomized clinical trial. JAMA 2016;315:2190-9.

16. Khwaja A. KDIGO clinical practice guidelines for acute kidney injury. Nephron 2012;120:179:c179-c184.

17. Klouche K, Gibney RTN, Forni LG. Can this patient be safely weaned from RRT? Intensive Care Med 2018;44:639-42.

18. Aniort J, Ait Hssain A, Pereira B, et al. Daily urinary urea excretion to guide intermittent hemodialysis weaning in critically ill patients. Crit Care 2016;20:43

19. Viallet $\mathrm{N}$, Brunot V, Kuster N, et al. Daily urinary creatinine predicts the weaning of renal replacement therapy in ICU acute kidney injury patients. Ann Intensive Care 2016;6:71.

20. Yong Z, Pei X, Zhu B, et al. Predictive value of serum cystatin $C$ for acute kidney injury in adults: a meta-analysis of prospective cohort trials. Sci Rep 2017;7:41012.

21. Zhang Z, Lu B, Sheng X, et al. Cystatin C in prediction of acute kidney injury: a systemic review and meta-analysis. Am J Kidney Dis 2011;58:356-65.

22. Katagiri D, Doi K, Matsubara T, et al. New biomarker panel of plasma neutrophil gelatinase-associated lipocalin and endotoxin activity assay for detecting sepsis in acute kidney injury. J Crit Care 2013;28:564-70.

23. Kim CS, Bae EH, Ma SK, et al. A prospective observational study on the predictive value of serum Cystatin $C$ for successful weaning from continuous renal replacement therapy. Kidney Blood Press Res 2018;43:872-81.

24. Yang T, Sun S, Zhao Y, et al. Biomarkers upon discontinuation of renal replacement therapy predict 60-day survival and renal recovery in critically ill patients with acute kidney injury. Hemodial Int 2018;22:56-65.

25. Zhang $\mathrm{Z}, \mathrm{Xu} \mathrm{X}, \mathrm{Ni} \mathrm{H}$, et al. Serum cystatin $\mathrm{C}$ is associated with renal function recovery in critically ill patients undergoing continuous renal replacement therapy. Nephron Clin Pract 2012;122:86-92.

26. Higgins JPT. Cochrane handbook for systematic reviews of interventions, 2011.

27. Moher D, Shamseer L, Clarke M, et al. Preferred reporting items for systematic review and meta-analysis protocols (PRISMA-P) 2015 statement. Syst Rev 2015;4:148-60.

28. Sampson M, McGowan J, Cogo E, et al. An evidence-based practice guideline for the peer review of electronic search strategies. J Clin Epidemiol 2009;62:944-52.

29. Higgins JP, Altman DG, Gøtzsche PC, et al. The Cochrane Collaboration's tool for assessing risk of bias in randomised trials. BMJ 2011;343:d5928-93.

30. Wells GA SB, O'Connell D. The newcastle-ottawa scale (NOS) for assessing the quality of nonrandomised studies in meta-analyses ottawa. ON: Ottawa: Hospital research institute, 20112016.

31. Stroup DF, Berlin JA, Morton SC, et al. Meta-analysis of observational studies in epidemiology: a proposal for reporting. JAMA 2000;283:2008-12.

32. Reitsma JB, Glas AS, Rutjes AW, et al. Bivariate analysis of sensitivity and specificity produces informative summary measures in diagnostic reviews. J Clin Epidemiol 2005;58:982-90. 